\title{
Anterior rhinoscopy and middle meatal culture in acute rhinosinusitis
}

\author{
U THUNBERG, K ENGSTRÖM, S OLAISON, S HUGOSSON \\ Department of Oto-rhino-laryngology, Örebro University Hospital and Örebro University, Sweden
}

\begin{abstract}
Objective: To assess the use of bacterial culture findings for middle meatal samples obtained via anterior rhinoscopy, in the diagnosis of adults with acute rhinosinusitis.

Materials and methods: Microbial cultures were prepared for 30 adult patients with acute rhinosinusitis and suspected bacterial involvement, using samples from the nasopharynx, and from the nasal middle meatus obtained via anterior rhinoscopy. Findings for the ipsilateral maxillary antrum were used as a reference.

Results: Seventeen patients had a bacterial infection as verified by a positive culture from the maxillary antrum. Middle meatal samples had a similar sensitivity but a better specificity, positive predictive value and negative predictive value, compared with nasopharyngeal samples, although predictive values were not statistically significant at a 95 per cent confidence level.

Conclusion: Anterior rhinoscopy with culture of middle meatal samples can be recommended as a diagnostic procedure for acute rhinosinusitis. The results can also guide the decision on antibiotic treatment.
\end{abstract}

Key words: Sinusitis; Bacteriology; Microbiology; Diagnosis

\section{Introduction}

Primary care physicians and otolaryngologists often examine and treat patients with acute rhinosinusitis. Acute rhinosinusitis is one of the most frequently made diagnoses in adults. In European populations, the incidence is reported to be $20-60$ per 1000 per year, according to the 2012 update of the European position paper on rhinosinusitis and nasal polyps. ${ }^{1,2}$

A bacterial involvement is often suspected, and the physician's main management decision is whether to treat with antibiotics or not. Despite the European position paper guidelines, this decision can be difficult to make.

A computed tomography (CT) scan can prompt suspicion of a bacterial infection if there is complete opacification of the antrum, or a fluid level. Sinus ultrasonography can detect secretion in the maxillary antrum, and provides an easy and non-irradiating alternative for this purpose. ${ }^{3}$

The recommended method of confirming bacterial rhinosinusitis is a positive culture from aspirated fluid from the maxillary antrum, after puncture through the nasal cavity beneath the concha inferior. However, this method is only acknowledged by otolaryngologists and is not suitable for routine diagnosis in primary care. $^{3,4}$

In primary care, nasopharyngeal cultures are common. However, nasopharyngeal culture has been proven to correlate inadequately with results from aspirated sinus secretions. ${ }^{5}$ Other studies have shown that middle meatal cultures are a good alternative, using an endoscope to obtain the specimen. ${ }^{6,7}$ Animal studies have shown a good correlation between culture findings in the middle meatus and the maxillary antrum. ${ }^{8}$

In primary care, the physician is usually confined to anterior rhinoscopy - nasal endoscopes are rarely available. Otolaryngologists routinely use anterior rhinoscopy in the initial assessment of the nasal cavity of patients with acute rhinosinusitis. It is an easy first line of examination and should always be performed for such patients. ${ }^{1}$

In light of the above, the present study aimed to establish the correlation between culture findings for the middle meatus, obtained via anterior rhinoscopy, and culture findings for maxillary antrum secretions, and to assess whether this correlation was better than that between culture findings for the nasopharynx and the maxillary antrum. 


\section{Materials and methods}

\section{Study participants}

Adult patients, i.e. 18 years or older, were recruited from the ENT department of Örebro University Hospital, Sweden. Patients were included if they had discoloured discharge from the middle meatus on anterior rhinoscopy, or complete opacification or a fluid level on radiological examination, and 2 or more of the following symptoms: congested nose, nasal secretion, facial pressure or pain, and loss of smell for more than 10 days but less than 3 months. ${ }^{1,2}$

Patients with maxillary sinusitis of suspected dental origin were excluded.

Information about previous sinus disease and ongoing antibiotic treatment was obtained. Ongoing antibiotic treatment was defined as antibiotic medication on the day of bacterial cultures or antibiotic treatment completed less than 2 days prior to cultures.

Most of the patients had been referred to the ENT department by their primary care physician, but four were emergency cases.

\section{Culture samples}

Culture samples were obtained from each subject's nasopharynx using a thin, cotton-tipped aluminium swab threaded through the nasal cavity.

The concha media and middle meatus were visualised by anterior rhinoscopy with a head lamp as the light source. In most cases, a nasal vasoconstrictive spray was administered, before obtaining the culture sample from the middle meatus using the same type of swab used in the nasopharynx.

After topical anaesthesia, a needle was used to puncture the ipsilateral maxillary antrum via the nasal cavity, beneath the concha inferior, and secretion was aspirated into a syringe. If no secretion was obtained, a few millilitres of saline were instilled into the sinus and aspirated after approximately 1 minute.

Samples from the nasopharynx and the middle meatus were transported to the laboratory in transport medium. The maxillary antrum sample was transported in the aspiration syringe.

\section{Laboratory analysis}

Samples from all three locations were cultured on blood agar medium (4.25 per cent Columbia II agar) and on blood agar medium incorporating 0.5 per cent gentian violet.

The maxillary antrum samples were also cultured on gonococcal (also termed GC) agar (gonococcal medium base, 3.6 per cent; Difco, BD Diagnostic Systems, Sparks, Maryland, USA), on mannitol agar (mannitol salt, 11.1 per cent; BBL, BD Diagnostic Systems) and in fastidious anaerobe broth with glucose 1 per cent. Samples were incubated for 2-7 days at $37^{\circ} \mathrm{C}$.

Bacterial type was identified according to the routine diagnostic procedures of the clinical bacteriology section of the laboratory medicine department, Örebro University Hospital. The finding of coagulase-negative staphylococci in all three locations was considered to represent contamination, and was recorded as equivalent to a negative culture finding.

Radiological findings were also documented.

\section{Ethical considerations}

The study subjects gave informed consent to their participation. The study was approved by the Regional Ethics Review Board, Uppsala.

\section{Statistical analysis}

A power calculation was made using the matched-pairs design in the case of binary responses. Based on our clinical experience, we made the assumption that culture findings for nasopharyngeal samples and for maxillary antrum samples should have 40 per cent agreement, and that culture findings for middle meatal samples and for maxillary antrum samples should have approximately 80 per cent agreement. Consequently, 30 individuals were required in order to detect a difference between the two test methods with at least 80 per cent power.

The 95 per cent confidence intervals for the test methods' sensitivity, specificity and predictive values were calculated with binomial distribution using Stata ${ }^{\circledR}$ software.

\section{Results}

Thirty patients were included, 6 men and 24 women. The age range was 21 to 81 years, with a mean age of 45 years.

Radiological investigations were performed on 29 patients, using plain X-ray or CT scans. In all examinations, the affected sinus showed complete opacification or a fluid level. One patient (number 16) received no radiological examination.

The culture findings for the nasopharynx, ipsilateral middle meatus and maxillary antrum are displayed in Table I. In 17 patients, bacterial infection was verified by a positive antral culture. The antibiotic sensitivity of these isolates was analysed. There were five cases caused by pneumococci, all with normal sensitivity to penicillin V. None of the seven Haemophilus influenzae strains produced beta-lactamase, and all three cases with Staphylococcus aureus were sensitive to isoxa-penicillin. Thirteen patients had ongoing antibiotic treatment. Bacterial growth was present in the maxillary antrum in seven cases despite ongoing antibiotic treatment at the time of sinus aspiration and culture. Four patients treated with penicillin $\mathrm{V}$ had $H$ influenzae on maxillary antrum culture, one patient with Streptococcus pneumoniae had ongoing treatment with amoxicillin, one patient with $S$ aureus had ongoing treatment with amoxicillin and clavulanic acid, and one patient with Moraxella catarrhalis had ongoing treatment with doxycycline. In 13 patients, the maxillary antrum culture was negative; one of 


\begin{tabular}{|c|c|c|c|c|c|c|}
\hline & & & $\mathrm{CU}$ & $\begin{array}{l}\text { TABLE I } \\
\text { URE RESULTS }\end{array}$ & & \\
\hline Pt no & Sex & Age $(y)$ & Maxillary antrum aspirate & & red organism \& growt & \\
\hline & & & & Nasopharynx & Middle meatus & Maxillary antrum \\
\hline 1 & $\mathrm{~F}$ & 21 & Mucopurulent & $\begin{array}{l}H \text { influenzae }++ \\
S \text { pneumoniae }+\end{array}$ & - & - \\
\hline 2 & $\mathrm{~F}$ & 35 & Purulent & M catarrhalis + & - & - \\
\hline 3 & $\mathrm{~F}$ & 37 & Mucopurulent & $S$ aureus + & $\mathrm{CNS}+$ & - \\
\hline 4 & M & 26 & Mucous & - & - & $\mathrm{CNS}+$ \\
\hline 5 & $\mathrm{~F}$ & 59 & Purulent & - & - & $M$ catarrhalis + \\
\hline 6 & M & 22 & Purulent & H influenzae + & $\mathrm{CNS}+$ & - \\
\hline 7 & $\mathrm{~F}$ & 25 & Mucous & - & - & - \\
\hline 8 & $\mathrm{~F}$ & 26 & Purulent & S pneumoniae ++ & $S$ pneumoniae +++ & $S$ pneumoniae +++ \\
\hline 9 & $\mathrm{~F}$ & 24 & Purulent & $M$ catarrhalis + & - & M catarrhalis + \\
\hline 10 & $\mathrm{~F}$ & 29 & Mucopurulent & H influenzae + & H influenzae + & $H$ influenzae +++ \\
\hline 11 & M & 35 & Purulent & - & $\mathrm{CNS}+$ & H influenzae +++ \\
\hline 12 & $\mathrm{~F}$ & 47 & Seromucous & $S$ pneumoniae + & $S$ pneumoniae + & $S$ pneumoniae + \\
\hline 13 & $\mathrm{~F}$ & 50 & Purulent & - & - & - \\
\hline 14 & M & 75 & Serous & $H$ influenzae +++ & $H$ influenzae +++ & - \\
\hline 15 & $\mathrm{~F}$ & 62 & Purulent & $\mathrm{CNS}+$ & - & - \\
\hline 16 & $\mathrm{~F}$ & 78 & Purulent & H influenzae + & H influenzae +++ & H influenzae +++ \\
\hline 17 & $\mathrm{~F}$ & 23 & Purulent & - & S pneumoniae ++ & $S$ pneumoniae +++ \\
\hline 18 & $\mathrm{~F}$ & 41 & None & - & - & - \\
\hline 19 & $\mathrm{~F}$ & 59 & None & $P$ aeruginosa + & - & - \\
\hline 20 & $\mathrm{~F}$ & 81 & Purulent & $S$ aureus +++ & $S$ aureus +++ & $S$ aureus +++ \\
\hline 21 & M & 62 & Mucopurulent & - & - & - \\
\hline 22 & $\mathrm{~F}$ & 61 & Purulent & - & - & $H$ influenzae +++ \\
\hline 23 & $\mathrm{~F}$ & 42 & Mucous & $H$ influenzae ++ & $H$ influenzae ++ & H influenzae + \\
\hline 24 & $\mathrm{~F}$ & 46 & Purulent & - & - & $H$ influenzae + \\
\hline 25 & $\mathrm{~F}$ & 34 & Purulent & $\mathrm{CNS}+$ & $\mathrm{CNS}+$ & - \\
\hline 26 & $\mathrm{~F}$ & 46 & None & H influenzae +++ & $H$ influenzae ++ & H influenzae + \\
\hline 27 & $\mathrm{~F}$ & 60 & Purulent & $S$ pneumoniae ++ & S pneumoniae + & $S$ pneumoniae +++ \\
\hline 28 & $\mathrm{~F}$ & 37 & Purulent & - & $\mathrm{CNS}+$ & S aureus + \\
\hline 29 & $\mathrm{~F}$ & 49 & Purulent & - & - & S pneumoniae + \\
\hline 30 & M & 64 & Purulent & $S$ aureus + & $S$ aureus + & S aureus + \\
\hline
\end{tabular}

Bacterial growth levels: $+=$ minor; $++=$ moderate; $+++=$ abundant; $-=$ none. Pt no $=$ patient number; $y=$ years; $F=$ female; $\mathrm{M}=$ male; $\mathrm{CNS}=$ coagulase-negative staphylococci

TABLE II ACCURACY OF TEST METHODS*

\begin{tabular}{|c|c|c|c|c|c|c|c|c|}
\hline \multirow[t]{2}{*}{ Site } & \multicolumn{2}{|c|}{ Sensitivity } & \multicolumn{2}{|c|}{ Specificity } & \multicolumn{2}{|c|}{ PPV } & \multicolumn{2}{|c|}{ NPV } \\
\hline & $n(\%)$ & $95 \% \mathrm{CI}^{\dagger}$ & $n(\%)$ & $95 \% \mathrm{CI}^{\dagger}$ & $n(\%)$ & $95 \% \mathrm{CI}^{\dagger}$ & $n(\%)$ & $95 \% \mathrm{CI}^{\dagger}$ \\
\hline Nasopharynx & 10/17 (59) & $33-82$ & $7 / 13(54)$ & $25-81$ & $10 / 16(62)$ & $35-85$ & $7 / 14(50)$ & $23-77$ \\
\hline Middle meatus & $10 / 17$ (59) & $33-82$ & $12 / 13(92)$ & $64-100$ & $10 / 11(90)$ & $59-100$ & $12 / 19(63)$ & $38-84$ \\
\hline
\end{tabular}

${ }^{*}$ Compared with maxillary antrum culture as the reference method. ${ }^{\dagger} 95 \% \mathrm{CI}=95 \%$ confidence interval of percentages. PPV $=$ positive predictive value; NPV = negative predictive value

these cultures grew coagulase-negative staphylococci. Six of these patients were receiving ongoing antibiotic treatment and seven were untreated. Patients with positive maxillary antrum cultures did not differ in their clinical appearance from patients with negative maxillary antrum cultures.

Previous episodes of acute sinusitis were reported by 23 patients (77 per cent). None of the patients experienced any complication of the disease, and all recovered without developing chronic inflammation of the sinuses.

The sensitivity, specificity, and positive and negative predictive values of the two test methods (i.e. culture from the nasopharynx and from the middle meatus) are presented in Table II, with the maxillary antrum culture findings used as a reference. There was no statistically significant difference between the two test methods regarding sensitivity, specificity, or positive or negative predictive values.

\section{Discussion}

It is important to try to identify the bacterial cause of acute rhinosinusitis in order to offer correct antibiotic treatment and to avoid antibiotics in cases that are of non-bacterial origin. To avoid the development of antibiotic resistance, the treatment should be well motivated with a microbial spectrum which is as narrow and accurate as possible. 
Symptomatic treatment for pain and for nasal congestion (with intranasal decongestants and/or corticosteroids) is also often indicated.

Antibiotic treatment is primarily indicated for patients who have severe facial pain, fever or a threatening complication of rhinosinusitis, but it can also be considered in patients with moderate symptoms if a bacterial infection is verified. ${ }^{1,9,10}$ The benefits of antibiotic treatment in cases of acute bacterial rhinosinusitis with moderate symptoms must be weighed against the potential for adverse effects. ${ }^{1,9}$

In the present study, despite the identification of a fluid level or complete opacification on radiological examination, 13 patients had no bacterial growth from maxillary antrum samples. Six of them were receiving ongoing antibiotic treatment; considering the possibility of a laboratory artefact, these patients may still have had a bacterial infection. However, at least 23 per cent $(7 / 30)$ had a non-bacterial rhinosinusitis and would have benefitted from symptomatic treatment only.

The sensitivity of the cultured pneumococcal and $H$ influenzae strains to penicillins is encouraging, and reflects the low prevalence of antibiotic resistance found in airway pathogens in Scandinavia. ${ }^{11,12}$

To our best knowledge, the present study is the first to correlate culture findings for the nasopharynx, and for the nasal middle meatus sampled via anterior rhinoscopy, with those for the maxillary antrum. The results indicate that bacterial findings for the maxillary antrum correlate slightly better with those for middle meatal secretions than those for the nasopharynx. Nasopharyngeal cultures had more false positive results (6 of 16) than middle meatal cultures ( 1 of $11)$, which is reflected in the better positive predictive value and specificity for middle meatal testing. Thus, it appears that nasopharyngeal specimens are more likely to be contaminated by irrelevant bacteria in the nasal vestibulum, compared with middle meatal specimens collected by anterior rhinoscopy.

Negative culture findings for the maxillary antrum, interpreted as non-bacterial rhinosinusitis, were also better identified by middle meatal sampling than nasopharyngeal sampling, reflected in the comparatively advantageous specificity and negative predictive value of middle meatal sampling. However, there were false negative results for both methods: 7 of 14 for nasopharyngeal samples and 7 of 19 for middle meatal samples. Ongoing antibiotic treatment in 13 patients should have affected culture findings for all three locations, and was not considered to alter the correlations between results for the maxillary antrum versus the two test methods.

The sensitivity of both test methods in detecting bacterial rhinosinusitis was 0.59 ; this was inferior to the reported sensitivity of sampling from the middle meatus as visualised by rigid endoscopy, ${ }^{6}$ a technique which should be preferred if available. One is more likely to reach the ostial area with the swab when using endoscopy compared with anterior rhinoscopy. Thus, endoscopic swabbing of the middle meatus appears to enable culture sample collection that more sensitively detects microbes in the maxillary antrum, compared with middle meatal sampling via anterior rhinoscopy.

The reference method of aspirating secretions from the maxillary antrum for culture should still be in use by otolaryngologists. It is the most accurate way of detecting relevant pathogens, and the collection method makes it possible to treat the patient's infection by antral irrigation and drainage at the same time. ${ }^{5,13}$ However, the benefits of antral irrigation and drainage in acute rhinosinusitis were questioned in the 2012 European position paper, ${ }^{1}$ and more studies are needed to elucidate the effects of this treatment option.

It is important to note that predictive values are influenced not only by the quality of the test method but also by the prevalence of the condition studied - in this study, bacterial rhinosinusitis, confirmed with positive maxillary antrum culture. ${ }^{14}$ The difference between the two test methods was not statistically significant at a 95 per cent confidence level. A significant difference may have been detected with a larger study population. However, there was a trend towards better diagnostic quality for middle meatal sampling via anterior rhinoscopy, compared with nasopharyngeal sampling, in detecting a bacterial cause of acute rhinosinusitis. Anterior rhinoscopy, preceded by the administration of a local vasoconstrictive spray, is still the first line of examination used by otolaryngologists, and general practitioners should be encouraged to perform this examination.

- Acute rhinosinusitis patients were studied

- Maxillary antrum culture results correlated slightly better with middle meatal results (sampling via anterior rhinoscopy) than with nasopharyngeal results

- The sensitivity of both latter methods was inferior to middle meatal culture via rigid endoscopy

- In the absence of rigid endoscopy, middle meatal sampling via anterior rhinoscopy is recommended

- Results can establish the microbial diagnosis and inform antibiotic treatment decisions

In cases of acute rhinosinusitis, a reasonable treatment regime would use symptomatic medication if there are mild or moderate symptoms and no threatening or manifest complications. In doubtful cases, the decision to treat with antibiotics or not can wait until the results of bacterial culture are available. If the culture is negative, symptomatic medication should be continued. Only if there is a positive middle meatal culture and persistent or worsening symptoms should antibiotic 
treatment be prescribed. A CT or ultrasound examination may be of additional help in making this decision.

\section{Conclusion}

In the search for a bacterial cause of acute rhinosinusitis, the present study findings indicate that culture sampling from the middle meatus via anterior rhinoscopy is preferable to standard nasopharyngeal culture, and that this procedure can be recommended to primary care physicians and otolaryngologists alike. When diagnosing acute rhinosinusitis, anterior rhinoscopy should be regarded as the first line of examination of the nasal cavity, and should precede the decision to treat with antibiotics or not; middle meatal culture sampling is easily performed at the same time.

\section{Acknowledgement}

The authors thank Anders Magnusson, Department of Epidemiology and Biostatistics, Örebro University Hospital, for his kind help with the statistical calculations.

\section{References}

1 Fokkens W, Lund VJ, Mullol J, Bachert C, Alobid I, Baroody F et al. EPOS 2012: European position paper on rhinosinusitis and nasal polyps 2012. Rhinology 2012;50(suppl 23):1-299

2 Fokkens W, Lund V, Bachert C, Clement P, Hellings P, Holmstrom $\mathrm{M}$ et al. EAACI position paper on rhinosinusitis and nasal polyps executive summary. Allergy 2005;60:583-601

3 Teppo H, Revonta M. Ultrasound device helps rule out maxillary sinus fluid in acute rhinosinusitis: how we do it. Clin Otolaryngol 2011;36:491-513

4 Varonen H, Rautakorpi UM, Huikko S, Honkanen PO, Klaukka T, Laippala P et al. Management of acute maxillary sinusitis in Finnish primary care. Results from the nationwide MIKSTRA study. Scand J Prim Health Care 2004;22:122-7
5 Berg O, Carenfelt C, Kronvall G. Bacteriology of maxillary sinusitis in relation to character of inflammation and prior treatment. Scand J Infect Dis 1988;20:511-16

6 Vogan JC, Bolger WE, Keyes AS. Endoscopically guided sinonasal cultures: a direct comparison with maxillary sinus aspirate cultures. Otolaryngol Head Neck Surg 2000;122:370-3

7 Araujo E, Palombini BC, Cantarelli V, Pereira A, Mariante A. Microbiology of middle meatus in chronic rhinosinusitis. $\mathrm{Am} \mathrm{J}$ Rhinol 2003;17:9-15

8 Vaidya AM, Chow JM, Stankiewicz JA, Young MR, Mathews HL. Correlation of middle meatal and maxillary sinus cultures in acute maxillary sinusitis. Am J Rhinol 1997;11:139-43

9 Ahovuo-Saloranta A, Rautakorpi UM, Borisenko OV, Liira H, Williams JW, Mäkelä M. Antibiotics for acute maxillary sinusitis. Cochrane Database Syst Rev 2010;(2)CD000243

10 Ahuja GS, Thompson J. What role for antibiotics in otitis media and sinusitis? Postgrad Med 1998;104:93-9

11 Lindbaek M, Melby KK, Schoyen R, Hjortdahl P. Bacteriological findings in nasopharynx specimens from patients with a clinical diagnosis of acute sinusitis. Scand $J$ Prim Health Care 2001;19:126-30

12 Molstad S, Erntell M, Hanberger H, Melander E, Norman C, Skoog $\mathrm{G}$ et al. Sustained reduction of antibiotic use and low bacterial resistance: 10-year follow-up of the Swedish Strama programme. Lancet Infect Dis 2008;8:125-32

13 Engquist S, Lundberg C, Venge P. Effects of drainage in the treatment of acute maxillary sinusitis. Acta Otolaryngol 1983; 95:152-9

14 Altman DG, Bland JM. Statistics notes: diagnostic tests 2: predictive values. BMJ 1994;309:102.1

Address for correspondence:

Dr Svante Hugosson,

Department of Oto-rhino-laryngology,

Örebro University Hospital and Örebro University,

SE-701 85 Örebro, Sweden

Fax: +4619103301

E-mail: svante.hugosson@orebroll.se

Dr S Hugosson takes responsibility for the integrity

of the content of the paper

Competing interests: None declared 